\title{
American Name Society Membership and Subscription Roster 196r
}

This list includes names and addresses of all individual and institutional members as of July 1, 1961.

Members and subscribers who have not paid the dues for 1961 are urgently requested to do so immediately in order to insure the uninterrupted delivery of Names.

Special interest in names is indicated by the following symbols:

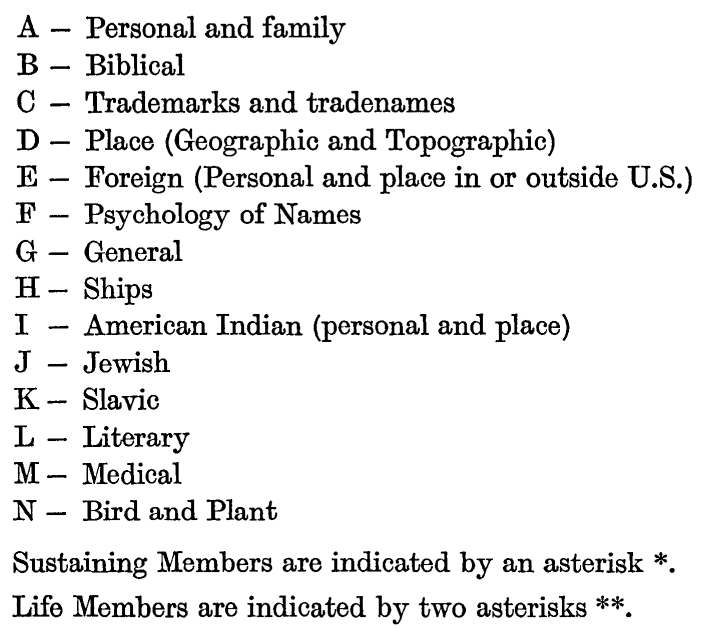

\section{INDIVIDUAL MEMBERS}

Patricia Anna Abel, 3302 S. 17th, Lincoln, Nebr. (G)

Samuel H. Abramson, 294 Fisher Ave., White Plains, N.Y. (EJ)

*Henry E. Abt, Brand Names Foundation, 437 Fifth Ave., New York 16, N.Y.

Louise Ackerman, 333 West Calvert, Lincoln, Nebr. (G)

Philip Adams, 1020 Mills Bldg., 220 Montgomery St., San Francisco 4, Calif.

Curtis Adler, 6053 Bellingham Ave., North Hollywood, Calif.

James E. Alatis, 5108 Sutton Place, Alexandria, Va.

Harold B. Allen, 317 Folwell Hall, Univ. of Minnesota, Minneapolis 14, Minn.

Morse S. Allen, 3 Fern St., Hartford 5, Conn. (D-Conn.)

John Q. Anderson, English, Texas A. and M., College Station, Texas

*Harry S. Ashmore, Editor-in-chief, Encyclopaedia Britannica, Box 4068, Santa Barbara, Calif.

William E. Ashton, 712 Fifth Ave., Helena, Mont. (D) 
E. Bagby Atwood, English, Univ. of Texas, Austin, Tex.

Lt. Col. Cortland P. Auser, English, Air Force Academy, Colo.

Etholine Aycock, 614 Remington, Fort Collins, Colo.

Joseph M. Backus, 2702 Virginia St., Berkeley 9, Calif.

Donald Gay Baker, 8th Ave., Collegeville, Pa. (D-Classical and N.H.)

**Clarence L. Barnhart, 141 Parkway Rd., Bronxville 8, N.Y.

Robert K. Barnhart, 355 S. State Rd., Briarcliff Manor, N.Y. (D)

S. V. Baum, 338 W. 88th St., New York 24, N.Y. (AC)

Richard Beck, Language, Univ. of North Dakota, Grand Forks, N.Dak.

Madison S. Beeler, 2413 Dwinelle Hall, Univ. of California, Berkeley 4, Calif. (I)

Mildred R. Bennett, 329 N. Cedar St., Red Cloud, Nebr. (L-Cather)

A. I. Bereskin, Controller of Surveys, Dept. of Natural Resources, Regina, Sak., Canada

Laura E. Berge, Lisco, Nebr.

Arthur Berliner, 1603 Macombs Rd., New York 52, N.Y. (D-NY Streets)

George Bijur, Guaranty Trust Co., Place Vendôme, Paris, France

Charles D. Blaney, 314 S. Water St., Crawfordsville, Ind.

Rev. J. T. Blanton, 2418 State St., Durham, N.C. (B)

William Clark Bliven, 523 Chestnut St., Grand Forks, N.Dak.

Bertha E. Bloodworth, 502 Hermitage Dr., Florence, Ala.

Mrs. Jeannette G. Blumengarten, 324 Ave. I, Brooklyn 30, N.Y. (D-NYC)

J. Boddewyn, Bus. Admin., Univ. of Portland, Portland 3, Ore.

Charles Boewe, 5 Balwyn Pl., Bala-Cynwyd, Pa.

Lalia Phipps Boone, 2027 NW 7th Lane, Gainesville, Fla.

Muriel Bowden, 115 E. 82nd St., New York 28, N.Y.

Walter P. Bowman, 5331 Baltimore Ave., Chevy Chase 15, Md.

F. W. Bradley, 4250 St. Clair Dr., Columbia 4, S.C.

Myron Brender, 114 W. 86 St., New York 24, N.Y. (F)

A. A. Brierly, Independence, Calif. (A-English origin)

Alan K. Brown, 25893 W. Fremont Ave., Los Altos Hills, Calif.

Samuel L. Brown, 2522 N.E. 4th St., Minneapolis 18, Minn. (AE)

Thomas P. Brown, 615 Alvarado St., San Francisco 14. Calif.

Edwin H. Bryan, Jr., B. P. Bishop Museum, Honolulu 17, Hawaii (AD-Hawaii, Pacific)

**Margaret M. Bryant, 1 Montague Terrace, Brooklyn 1, N.Y.

Warren H. Buell, 608 N. Cherokee Ave., Los Angeles 4, Calif. (G)

Meredith F. Burrill, 5503 Grove St., Chevy Chase 15, Md. (D)

Robert A. Caldwell, English, Univ. of North Dakota, Grand Forks, N.Dak. (LD)

Arthur L. Campbell, 5066 Romaine St., Los Angeles 29, Calif. (A)

John S. Caponnetto, 440 New Jersey Ave., N. W., Washington 1, D.C.

Mrs. Helen Carlson, English, Western Illinois Univ., Macomb, Ill. (D)

George Revilo Carter, 5540 Hyde Park Blvd., Chicago 37, Ill. (G)

Frederic G. Cassidy, 315 Bascom Hall, Madison 6, Wisc.

Mrs. Gerald (Ina Sizer) Cassidy, 924 Canyon Rd., House 5, Santa Fe, New Mexico

Ava H. Chadbourne, Box 14, MRB, Bangor, Me. (IP-Me)

Ben F. Cheek, Jr., P.O. Box 387, Toccoa, Ga.

William C. Childers, 207 Anderson Hall, Univ. of Florida, Gainesville, Fla. (L) 
Lewis H. Chrisman, 87 Smithfield St., Buckhannon, W.Va.

Donald H. Clark, 4329 Semple St., Seattle 99, Wash. (D-Wash)

Donald T. Clark, 25 Moon Hill Rd., Lexington 73, Mass.

M. Graham Clark, School of the Ozarks, Point Lookout, Mo.

Robert L. Coard, Lang. \& Lit., St. Cloud State College, St. Cloud, Minn. (L)

D. B. Cofer, Box 325, College Station, Tex. (A)

Harry C. Coffin, Geography, Arizona State Univ., Tempe, Ariz.

Hennig Cohen, American Studies Assoc., Box 46, College Hall, Univ. of Pennsylvania, Philadelphia 4, Pa. (D)

Charles Collins, 716 Brummel St., Evanston, Ill. (G)

Henry Q. Collins, 154 N. Portsside, Chicago 44, Ill.

Ernest E. Cook, 10354 Mississippi Blvd., Coon Rapids, Minn.

Joseph N. Corcoran, 1304 Philadelphia National Bank Bldg., Philadelphia 7, Pa. Ephraim Cross, 215 W. 98th St., New York 25, N.Y. (EJ-Chinese, Indo-European)

Thomas L. Crowell, 15 Claremont Ave., New York 27, N.Y.

Marvin E. Cruse, 847 Bellevue Ave., Hastings, Nebr.

T. J. Curran, 1235 Fairfield Rd., Victoria, British Columbia, Canada

Jack A. Dabbs, 1011 Edgewood, Bryan, Tex. (GE-Latin Am)

Dr. Moshe Davis, 39 Ramban St., Jerusalem, Israel

G. F. Delaney, 1701 Ainsley Dr., Ottawa 3, Ont., Canada

Roland Dickison, Arkansas College, Batesville, Aik. (AD)

Elliott V. K. Dobbie, Philosophy Hall, Columbia Univ., New York 27, N.Y.

B. W. Dombrowski, St. John's College, Univ. of Manitoba, Winnipeg 9, Canada Mathurin Dondo, P.O. Box 213, Wailuku, Mauri, Hawaii (G)

John Richard Drake, Jr., 1923 Mary Catherine, Ypsilanti, Mich.

Robert T. Drake, 711 Sheridan Rd., Winnetka, Ill.

Henri Draye, Winkselse Steenweg 60, Herent, Louvain, Belgium

Geart Droege, 5112 Broadway, Chicago 40, Ill. (E-Frisian and Netherlands)

Paul M. Dubbs, 163 East Curtin St., Bellefonte, Pa.

Audrey R. Duckert, English, Univ. of Massachusetts, Amherst, Mass. (D)

R. M. Duncan, Univ. of New Mexico, Albuquerque, N.Mex. (E-Hispanic)

A. R. Dunlap, English, Univ. of Delaware, Newark, Del. (D-Del)

Karl W. Dykema, Youngstown Univ., Youngstown, 0.

J. Harold Easterby, S. Car. Archives Dept., 1430 Senate St., Columbia, S.C.

S. J. Eckerson, General Drafting Co., Convent Station, N.J.

Edith F. Edward, 7118 East End Ave., Chicago 49, Ill. (D)

Edward C. Ehrensperger, Univ. of South Dakota, Vermillion, S.Dak. (D-S.Dak)

M. B. Emeneau, Linguistics, Univ. of California, Berkeley 4, Calif. (G)

Harry Engels, 717 Powell Ave., Town of Mt. Royal, Montreal, Canada

E. E. Ericson, 1811 Grande Ave., S.E., Cedar Rapids, Iowa

Nathan L. Eudy, 1933 E. Geer St., Durham, N.C. (A)

G. Thomas Fairclough, 3103 Saint Paul St., Baltimore 18, Md.

Herbert G. Fales, 67 Wall St., New York 5, N.Y. (G)

*Francis P. Farquhar, 2930 Avalon Ave., Berkeley .5, Calif.

John H. Fawcett, 2669 Shasta Rd., Berkeley 8, Calif. (G)

William Feather, 19201 Van Aken Blvd., Cleveland 22, O. (G)

Thomas P. Field, Geography, Univ. of Kentucky, Lexington, Ky. (DI-Ky) 
Nell Green Findley, Box 217, Canyon, Tex. (C-Chem)

W. Bruce Finnie, 116 East Tompkins St., Columbus 2, O. (D-OValley)

Edith M. Fitton, 104 Tenney Ct., Juniper Hills, Oxford, O. (D)

${ }^{*}$ C. S. Forester, 1066 Park Hills Rd., Berkeley, Calif.

Richard L. Forstall, P.O. Box 7600, Chicago 80, Ill. (D)

Joseph N. Frank, 28 Sunset Ave., Montreal 8, Quebec, Canada

Gösta Franzen, Faculty Exchange, Univ. of Chicago, Chicago 37, Ill.

Ethel Dunbar Froehlich, 2032 N. 36th St., Milwaukee 8, Wis.

Anming A. Fu, 5221 Nebraska Ave., N. W., Washington 15, D.C.

Joseph G. Fucilla, Romance Languages, Northwestern Univ., Evanston, Ill. (G)

*J. C. Furnas, R. D. 1, Lebanon, N.J.

Wilbur G. Gaffney, 121 Andrews Hall, Univ. of Nebraska, Lincoln, Nebr.

Rev. James A. Geary, 4306 28th Pl., Mt. Rainier, Md.

William J. Gedney, English, Univ. of Michigan, Ann Arbor, Mich.

Patrick J. M. Geelam, Royal Geographic Soc., 1 Kensington Gore, London SW 7, England

Ignace J. Gelb, Assyriology, Oriental Institute, Univ. of Chicago, Chicago 37, Ill.

(G-Typology, Ancient Oriental)

**Demetrius J. Georgacas, 2 Georgiou Vendiri, Filotheï, Athens, Greece (ADEIndo-European, Greek, Latin, Modern Greek)

William H. Gilbert, 9015 First Ave., Silver Spring, Md.

Charles Edgar Gilliam, 316 Union Trust Bldg., Petersburg, Va.

Joshua B. Glasser, 39 South LaSalle St., Chicago 3, Ill.

**C. M. Goethe, 3731 Tea St., Sacramento 16, Calif.

Philip B. Gove, G. \& C. Merriam Co., Springfield 2, Mass.

Mrs. Byrd H. Granger, English, Univ. of Arizona, Tucson, Ariz. (D-Ariz)

Wanda K. Green, Bloomfield, Colo.

John W. Greenfield, 7917 Poinsettia Dr., Pleasure Ridge Park, Ky.

W. Cabell Greet, 450 Riverside Dr., New York 27, New York

Ewald T. Grether, Graduate School of Bus. Admin., Univ. of California, Berkeley 4, Calif. (C)

Elisabeth K. Gudde, 137 Crest View Dr., Orinda, Calif.

David B. Guralnik (See World Publishing Co., Institution Roster)

George P. Hammond, 810 Contra Costa Ave., Berkeley 7, Calif.

Louise Hanley, 310 N. Livingston St., Madison 3, Wisc. (AD-Ky)

Kelsie B. Harder, Youngstown Univ., Youngstown, O. (GL)

John P. Harrington, 125 W. Carrillo St., Santa Barbara, Calif.

Jesse W. Harris, Southern Illinois Univ., Carbondale, Ill.

James D. Hart, 740 San Luis Rd., Berkeley 7, Calif.

A. A. Hartman, 1121 S. State St., Rm. 1004, Chicago 5, Ill. (F)

Edmund Heier, Univ. of Waterloo, Waterloo, Ontario, Canada

Atcheson L. Hench, English, Pavilion IX, West Lawn, Univ. of Virginia, Charlottesville, Va. (G)

Carl Hepp, 155 Elm Ave., Mill Valley, Calif.

Frederick W. Hilbig, Genealogist, 1149 Elgin Ave., Salt Lake City 6, Utah

Douglas P. Hinkle, English, Sweet Briar College, Sweet Briar, Va.

Norman D. Hinton, English, 22 McCosh, Princeton Univ., Princeton, N.J. 
Robert Hitchman, 611 13th Ave., E., Seattle 2, Wash.

Catherine Horner, Apt. 8, 58 W. 3rd N., Salt Lake City 16, Utah

Cecilia A. Hotchner, 579 John St., Teaneck, N.J. (A)

Per Hovda, General Birchgate 30, Oslo, Norway

Arthur H. Hughes, Trinity College, 73 Vernon St., Hartford 6, Conn. (D-Conn.)

Bertram L. Hughes, Sterling College, Sterling, Kan.

R. E. Isto, 520 Illinois St., Fairbanks, Alaska (D)

Herman Iventosch, 600 Burke St., Easton, Pa.

Helen P. Jackson, Hotel Californian, San Francisco 2, Calif.

John B. Jackson, Box 2149, Santa Fe, N.Mex. (G)

Assar Janzén, Univ. of Calif., Berkeley 4, Claif. (E-Ger)

Ph. N. Jenner, DAC, 115 Kuukama St., P. M. Kailua, Oahu, Hawaii

Kiichi Jimbo, 8758 Babasaki, Fuchu-Shi, Tokyo, Japan

E. Gustav Johnson, 3225 Foster Ave., Chicago 25, Ill.

Oscar F. Jones, 1130 S. W. 8th Ave., Gainesville, Fla.

Petras Jonikas, 281 Olmstead Rd., Riverside, Ill.

Kanji Kagami, 18-2, Aratacho, Showaku, Nagoya, Japan

Rabbi Benzion C. Kaganoff, 2901 W. Greenleaf, Chicago 45, Ill.

Henry R. Kahane, Univ. of Illinois, Urbana, Ill.

Walter Th. Kammann W., Apartado Postal 1891, Caracas, Venezuela

Henry Karpenstein, 80 Ord St., San Francisco 17, Calif.

Julian B. Kaye, 436 Eastern Parkway, Brooklyn 25, N.Y.

Allen B. Kellogg, 1411 Loretta Drive, Indianapolis 27, Ind. (AL)

Jean McClure Kelty, Youngstown Univ., Youngstown, O. (GL)

Mrs. Gerald D. Kennedy, Rt. 2, Box 975, Stockton, Calif. (G)

Hamill Kenny, Box 31, Mt. Rainier, Md. (ADI)

Col. Calvin I. Kephart, Box 52, Shady Side, Md. (ADIE-Sp)

B. B. Khleif, 3930 Clark St., Kansas City 11, Mo.

Robert Klymasz, 101 Cunnington Ave., Winnipeg 8, Manitoba, Canada

Helge Kökeritz, Yale Univ., New Haven, Conn.

Memas Kolaitis, 1319 F St., N.W., Suite 702, Washington 4, D.C.

David Kosh, 50 E. 79 St., New York 21, N.Y.

P. K. Kovaliv, 518 9th St., N.W., Apt. 410, Washington 2, D.C.

Walter C. Kraft, Modern Lang., Oregon State College, Corvallis, Ore.

Fritz L. Kramer, Geography, University of Oregon, Eugene, Ore. (D)

Henry Kratz, Foreign Lang., Univ. of Oregon, Eugene, Ore.

Krikor Krikorian, 2500 Wisconsin Ave., N.W., Washington 7, D.C.

John R. Krueger, Oriental Lang., Univ. of California, Berkeley 4, Calif.

Aneale L. Kushmar, 2735 Taylor, Detroit 6, Mich. (G)

Charlton G. Laird, 1450 Mallory Lane, Reno, Nev. (G)

Symington P. Landreth, 1 Pine Grove, Briston, Pa.

Joseph Lantieri, 327 E. 45th St., New York 22, N.Y.

Donald W. Lee, English, Univ. of Houston, Houston, Tex. (G)

Clare D'Artois Leeper, 1468 Ross Ave., Baton Rouge 8, La.

*John Leighly, 1435 Arch St., Berkeley 8, Calif.

Norman B. Levin, Languages, Univ. of North Dakota, Grand Forks, N.Dak. 
Harry L. Levy, 404 E. 66th St., New York 21, N.Y.

Mrs. Irene Marie Lewis, 3880 Nithsdale St., South Burnaby, B.C., Canada

Mrs. Arthur Liman, 50 W. 9th St., New York 11, N.Y.

Grant Loomis, 828 Coventry Rd., Berkeley 7, Calif. (G-Wordplay)

Robert W. Lowe, Modern Languages, Georgetown Univ., Washington, D.C.

Sandra Luger, 1233 Astor Ave., Bronx 69, New York

John Lyman, 7801 Gateway Blvd., Washington 28, D.C. (H)

W. L. McAtee, 3 Davie Circle, Chapel Hill, N.C. (N)

A. R. McCrady, 2285 Mar Vista, Altadena, Calif. (A)

John T. McCutcheon, Jr., "A Line of Type or Two," Chicago Tribune, Chicago 11, Ill. (D)

Raven I. McDavid, Jr., English, University of Chicago, Chicago 37, Ill.

John Francis McDermott, 6345 Westminster Pl., St. Louis 30, Mo. (G)

William A. McDonald, 1489 Hythe, St. Paul 8, Minn.

Kathryn A. McEuen, 1160 Ocean Ave., Brooklyn 30, New York

James G. W. MacLamroch, Box 1588, Greensboro, N.C. (A)

E. Wallace McMullen, 15 Rosewood Dr., Madison, N. J. (DG)

J. Tweed McMullen, Box 746, Clearwater, Fla. (G)

John McNamara, 90 B Edgewater Park, Bronx 65, N.Y.

Alexander McQueen, 5222 N. Lakewood, Chicago 40, Ill.

James M. McWilliams, 9 Arlmonte Dr., Berkeley 7, Calif. (G)

Ernest Maass, 150-76 Village Rd., Jamaica 32, New York (AJ)

Otto J. Maenchen, 10 Greenwood Common, Berkeley 8, Calif.

Elsie Mag, G. \& C. Merriam Co., Springfield 2, Mass.

Yakov Malkiel, 4333 Dwinell Hall, Univ. of California, Berkeley, Calif. (G-Romance)

**Kemp Malone, English, The Johns Hopkins Univ., Baltimore 18, Md.

Frederick Manfred, Blue Mound, Luverne, Minn. (L)

Albert H. Marckwardt, Univ. of Michigan, Ann Arbor, Mich.

Max Markrich, 248 15th Ave., San Francisco 18, Calif.

Joseph Martin, Jr., 400 Montgomery St., San Francisco 4, Calif. (G)

Warren R. Maurer, German, Univ. of California, Berkeley 4, Calif. (L)

**Mamie Meredith, 2340 Sumner St., Lincoln 2, Nebraska

Rev. Louis Mesko, 120 Rumsey Rd., Buffalo 9, New York

*Theodore R. Meyer, 3528 Washington St., San Francisco 18, Calif.

A. F. Michaelis, Standard Oil Co., 225 Bush St., Rm. 2174, San Francisco, Calif.

Josephine Miles, 2275 Virginia St., Berkeley 9, Calif.

Clarence W. Minkel, Colorado State College, Greeley, Colo. (DF-Latin Am)

Arthur Minton, R.F.D. 1, New Milford, Conn. (G)

Albert F. Moe, 4729 North Washington Blvd., Arlington 5, Va. (G)

Capt. Victor A. Moitoret, USN, Cinsusnaveur Staff Box 7, FPO New York, New York

G. M. Monroe (See Canadian Board on Geographical Names, Ottawa, Canada;

Institution Roster)

Maurice A. Mook, Boalsburg, Pa. (A-Ethnic and Social)

S. G. Morley, 2635 Etna St., Berkeley 4, Calif. (G)

Maralyn B. Morton, Idaho State College, Pocatello, Idaho (D-Idaho)

Armond Moyer, Rt. 2, Allentown, Pa.

Eugene V. Muench, New York Univ., 550 First Ave., New York 16, New York 
John G. Mutziger, 4711 Riverdale Rd., Riverdale, Md. (D)

J. Franklin Murray, SJ, English, Spring Hill College, Mobile, Ala. (D-Hesvit)

George K. Nakagawa, 901 Kokohead Ave., Honolulu, Hawaii

Samuel G. Naparstek, 2550 Independence Ave., New York 63, New York

Claude Henry Neuffer, English, Univ. of South Carolina, Columbia 1, S.C. (D-S. Car.)

Weldon N. Niva, 34 S. 18th St., Allentown, Pa. (C)

Delf Norona, 315 7th St., Moundsville, W.Va.

Benjamin Nunez, Georgetown Univ., Washington 6, D.C.

Robert D. Ochs, 15-D Cornell Arms, Columbia 1, S.C.

Petro Odarchenko, 2104 Newport Pl., N.W., Washington 7, D.C.

Clarence B. Odell, Denoyer-Geppert Co., 5235 Ravenswood Ave., Chicago 40, Ill. $(\mathrm{G})$

Joseph Orr, 105-38 63 Rd., Forest Hills 75, N.Y.

Allan H. Orrick, English, Rutgers Univ., New Brunswick, N.J.

Donald Jos. Orth, 2000 N. Upton St., Arlington, Va.

Marjorie O'Shaughnessy, 38 Park St., Montclair, N.J.

Donald R. Ostberg, Box 28, East Millstone, N.J.

Mrs. Ruth Sowers Owen, Box 57, Thomasville, N.C.

George B. Pace, English, Univ. of Missouri, Columbia, Mo.

William Paff, R. F. D. 1, Iowa City, Iowa (E-Germanic)

George C. Pappageotes, Box 48, Philosophy Hall, Columbia Univ., NY. 27, N.Y.

Oronzo Parlangeli, via G. Brunetti 51, Novoli (Lecce), Italy

Witold H. Paryski, Antalowka 7, Zakopane, Poland

John P. Pauls, 3422 Lyleburn Pl., Cincinnati 20, O. (K)

Ruth L. Pearce, 257 Cross Rd., Merion Station, Pa.

*T. M. Pearce, Univ. of New Mexico, Albuquerque, N.Mex. (GD-NMex)

Mario Pei, Columbia Univ., New York 27, N.Y. (AD)

Alfred Percy, Percy Press Publs., Elon Rd., Madison Heights, Va. (D)

Edwin N. Perrin, English, Dartmouth College, Hanover, N.Hamp.

Henry A. Person, Univ. of Washington, Seattle 5, Wash.

Emilio Peruzzi, Via Giambologna 10, Florence, Italy (G)

Henry Pettit, English, Univ. of Colorado, Boulder, Colo. (G)

Rev. Benedict Pfaller, OSB, Assumption Abbey, Richardton, N.Dak.

Charles A. Philhower, 303 Mountain Ave., Westfield, N.J.

Abraham Piianaia, 1938 Wilhelmina Rise, Honolulu 16, Hawaii

Winthrop W. Piper, 105 Egremont Ave., Pittsfield, Mass.

Robert Plank, 2387 Overlook Rd., Cleveland Heights 6, O. (F)

George H. Pollock, Institute Psychoanalysis, 664 N. Michigan Ave., Chicago 11, Ill. (F)

Roger Price, 275 Central Park West, New York 24, N.Y.

Newbell N. Puckett, Sociology, Western Reserve Univ., Cleveland 6, 0.

*Mrs. Delia H. Pugh, 130 W. Broad St., Burlington, N.J. (AD)

Ernst Pulgram, Romance Languages, Univ. of Michigan, Ann Arbor, Mich. (G)

Thomas Pyles, 629 Boulevard, Gainesville, Fla. (A)

Lili Rabel, English, Louisiana State Univ., Baton Rouge, La.

C. J. Raeth, Miami Univ., Oxford, O. (G) 
William Randel, English, Florida State Univ., Tallahassee, Fla. (D)

H. F. Raup, Geography \& Geology, Kent State Univ., Kent, O. (D)

Allen Walker Read, 39 Claremont Ave., New York 27, New York

P. H. Reaney, 35 Leigh Rd., Hildenborough, Tunbridge, Kent, England (D)

David W. Reed, English, Univ. of California, Berkeley 4, Calif.

George Rentz, Aramco Overseas Co., Box 1855, Cairo, Egypt

Mrs. R. D. Reser, 222 West Mulberry, San Antonio 12, Texas

Roger Revelle, Scripps Institution for Oceanography, La Jolla, Calif.

T. W. Reynolds, 312 Cascade Lane, Riviera Beach, Fla.

Melvin B. Ricks, Box 2524, Juneau, Alaska (D-Pacific Coast)

Roger A. Risley, R. R. 1, Box 430, Acton, Ind.

Stanley L. Robe, Spanish, University of California, Los Angeles 24, Calif. (E-Sp.)

A. H. Roberts, 1830 Helena St., Madison 4, Wisc.

Rev. John S. Roberts, 469 3rd St. W., Sonoma, Calif.

Kimberley S. Roberts, Modern Languages, Univ. of Delaware, Newark, Del.

(E-European)

R. D. Roberts, 315 Shearer Bldg., Bay City, Mich. (A)

Ernest M. Robson, R. D. 1, Kempton, Pa.

Zvonko R. Rode, 4100 W. St., N. W., Washington 7, D.C. (A-Etymology of Ancient Names)

P. Burwell Rogers, English, Bucknell Univ., Lewisburg, Pa.

Jose Pedro Rona, Republic de el Salvador 3284, Montevideo, Uruguay (EA)

Francis C. Rosenberger, 424 Senate Office Bldg., Washington, D.C.

Morris Rosenblum, 959 Carroll St., Brooklyn 25, New York

Mildred E. Ross, Box 94, Northwest Missouri State College, Maryville, Mo.

Stowell Rounds, Cannondale, Conn. (D-Road)

**J. B. Rudnyćkyj, Slavic Studies, Univ. of Manitoba, Winnipeg, Canada

F. G. Ruffner, 4212 Courville, Detroit 24, Mich.

I. Willis Russell, Mallard Lake, Rt. 2, Cottondale, Ala.

John Rydjord, 1730 N. Lorraine, Wichita 14, Kan. (D-Kan)

Harry J. Sadler, 3451 N.E. Lincoln St., Minneapolis 18, Minn.

Donald B. Sands, English, Boston College, Chestnut Hill 67, Mass.

Jacob Schauer, 2 West End Ave., Brooklyn 35, New York (G)

J. E. Schmidt, M. D., Charlestown, Ind. (M)

Arthur C. Schofield, 246 N. High St., Columbus 16, O. (G)

Mrs. Eva Schwartzman, 350 N. Milwaukee Ave., Libertyville, Ill. (G)

Richard B. Sealock, 5819 McGee St., Kansas City 13, Mo. (D-Biblio)

William M. Seaman, Foreign Languages, Michigan State College, East Lansing, Mich.

E. R. Seary, Memorial Univ. of Newfoundland, St. John's, Newfoundland, Canada (D)

Pauline A. Seely, 1050 Sherman St., Denver 3, Colo. (D-Biblio)

Charles L. Sellers, 621 Kennesaw Ave., Atlanta 8, Ga. (AD)

Alfred Senn, 207 Cynwyd Rd., Bala-Cynwyd, Pa. (E-Swiss and Lithuanian)

Rabbi Samson A. Shain, 508 N. Duke St., Lancaster, Pa. (BD-Pa)

George Pope Shannon, R. F. D. 6, Andalusia, Ala. (G)

Esther K. Sheldon, 352 Argyle Rd., Brooklyn 18, New York (G)

David Simon, 45-592C Paleka Rd., Kaneohe, Hawaii 
Yar Slavutych, Slavonic Div., Univ. of Alberta, Edmonton, Alberta, Canada (K) Alexander Sloan, 28 Overlook Terrace, Maplewood, N.J.

Joseph F. S. Smeall, 1116 S. 16th St., Grand Forks, N.Dak.

Elsdon C. Smith, 322 Sherman Ave., Evanston, Ill. (A)

Sidney Snook, Elizabethtown, Ill.

Lee L. Snyder, 341 W. Durham St., Philadelphia 19, Pa.

Robert Sonkin, City College 139th St. \& Convent Ave.,New York 31, New York (G)

Ralph W. Sorvig, Rt. 2, Box 44A, Fort Collins, Colo.

David G. Speer, Modern Lang., Purdue Univ., Lafayette, Ind.

Mrs. H. G. E. Spray, 15912 S. E. 8th St., Bellevue, Wash.

Gustavo Stahl, 10A Calle 6-13 Z 1, Guatemala City, Guatemala

Taylor Starck, Boylston Hall, Harvard Univ., Cambridge 38, Mass.

D. T. Starnes, 804 W. $301 / 2$ St., Austin 5, Tex.

W. A. Starr, 405 Montgomery St., San Francisco 4, Calif.

Anna Stearns, 4000 Beaconsfield Ave., Montreal 28, P. Q., Canada

Basil W. Steciuk, 63 Sussex St., Jersey City 2, N.J.

Vilhjalmur Stefansson, Dartmouth College Lib., Hanover, N.H.

Jess Stein, Random House, 457 Madison Ave., New York 22, N.Y. (G)

Mrs. Mary Florence Steiner, 527 Washington St., Wilmette, Ill. (E-Sp.)

Noel C. Stevenson, Box 1195, Wasco, Calif. (D-Calif.)

George R. Stewart, 100 Codornices Rd., Berkeley, Calif.

Sterling A. Stoudemire, 712 Gimghoul Rd., Chapel Hill, N.C. (E-Spanish)

O. Paul Straubinger, 4607 Oakwood Pl., Riverside, Calif.

Arval L. Streadbeck, SH 1560, Univ. of Utah, Salt Lake City 12, Utah (AD-Utah)

Sam Stulberg, 7311 73rd Ct., Hyattsville, Md.

Donald C. Swanson, 310 Folwell Hall. Univ. of Minnesota, Minneapolis 14, Minn. (GE)

Eleanor Symons, 917 Louisiana, Lawrence, Kan.

Churchill C. Sze, 1848 Columbia Rd., NW, Washington 9, D.C.

Walter S. Taintor, 60 Superior St., Oak Park, Ill. (A)

Ashley F. Talbot, 38 Winans St., East Orange, N.J.

*Peter Tamony, 2876 24th St., San Francisco 10, Calif.

Edward Taube, Univ. of Wisconsin, 620 Lake Ave., Racine, Wis.

*Thomas E. Taylor, U. S. G.S., Topo. Div., Federal Center Bldg. 25, Denver 2, Colo.

I. J. Tepperman, 548 Palmerston Blvd., Toronto, Canada

John A. Thomopoulos, Gymnasium, Syros, Greece

Laurence C. Thompson, Far Eastern Dept., Univ. of Washington, Seattle 5, Wash.

James A. Thorburn, Uniy. of Missouri, 8001 Natural Bridge Rd., Normandy 21, Mo. (AD)

Gutierre Tibón, Bucareli 48, Ciudad de Mexico

Richard A. Toerne, 60 E. 32nd, Apt. 902, Chicago 16, Ill.

B. L. Trippett, Advertising Dept., Nationąl Cash Register Co., Dayton 9, 0.

F. H. Trolle-Steenstrup, 6309 33rd St., NW, Washington 15, D.C.

Harry L. Tylor, 4427 Harcourt Rd., Baltimore 14, Md. (G)

Harlan L. Umansky, Emerson High School, Union City, N.J. (G)

Francis L. Utley, 164 W. 17th Ave., Columbus 14, O. (BG)

Kenneth H. Vanderford, Escalinata I, Madrid, Spain 
John A. Van Eerde, Romance Languages, Lehigh Univ., Bethlehem, Pa. (E-French) Michael S. Vartanoff, 6825 Wilson Lane, Bethesda 14, Md. (D)

Eugene B. Vest, Chicago Undergraduate Div., Univ. of Illinois, Chicago, Ill. (AD) *James M. Vicary, 22 E. 60th St., New York 22, N.Y.

Virgil J. Vogel, 11243 S. Homewood Ave., Chicago 43, Ill.

A. J. Walker, English, Georgia Institute of Tech., Atlanta 13, Ga. (AD-Atlanta streets)

Joseph A. Ward, Box 1013, Southwestern Louisiana Inst., Lafayette, La.

*R. Gordon Wasson, 23 Wall St., New York 8, New York

Harriet Weeks, 53 E. 96th St., New York 28, New York

C. A. Weslager, Old Public Rd., Hockessin, Del.

Helen P. Wheeler, Vassar College, Poughkeepsie, New York

J. R. Williams, La Marsa - Corniche, Tunisia

Margery M. Williams, 63 Whittredge Rd., Summit, N.J.

Andrew W. Wilson, Geography, Univ. of Arizona, Tucson, Ariz. (D)

Raymond R. Wilson, Box 471, Springfield 1, Mass. (A)

Thomas C. Wilson, 475 Arlington Ave., S., Reno, Nev.

John N. Winburne, 1450 N. Hagadorn Rd., East Lansing, Mich.

Henry B. Woolf, 127 School St., Springfield 5, Mass. (G)

Michael Woroby, 650 Machray Ave., Winnipeg 4, Manitoba, Canada

A. J. Wraight, Senate Courts, Apt. 107, 120 C St., NE, Washington, D.C. (D)

Charles L. Wrenn, Pembroke College, Oxford, England

Mrs. Bertha L. Wright, 71 Madison Ave., Mount Holly, N.J.

Thomas L. Wright, 6231 NE 154th St., Bothell, Wash.

Wilbur Zelinsky, Geography, Southern Illinois Univ., Carbondale, Ill.

John W. Zerkowski, M.D., 173 Meadowbrook Rd., Orchard Park, N.Y. (A)

Gertrude M. Zimmer, 221 Patterson St., Farmington, Mo. (D)

Edgar T. Zook, 1101 Alaska Commercial Bldg., 310 Samsome St., San Francisco 4, Calif.

\section{INSTITUTIONAL MEMBERS}

Air University Library, Maxwell AFB, Ala.

University of Alabama, Main Library, University, Ala.

University of Alaska Library, Box 1058, College, Alaska

Almanaque Americano, Apartado Postal 13665, Admn. 45, Mexico, F.D., Mexico

American Antiquarian Society, Park \& Salisbury, Worcester 5, Mass.

Arizona State University, Matthews Library, Tempe, Ariz.

Library, University of Arizona, Tucson, Ariz.

Arkansas College, State College, Ark.

University of Arkansas, General Library, Fayetteville, Ark.

Atlanta Public Library, 126 Carnegie Way, N.W., Atlanta 3, Ga.

Australian National University Library, Institute of Advanced Studies, Box 4,

G.P.O., Canberra, A.C.T., Australia

Institut für Völkerkunde, Wien 1, Reitschulgasse 2, Austria

Library, Ball State Teachers College, Muncie, Ind.

Bangor Public Library, Bangor, Me. 
Baylor University Library, Box 6307B, University Station, Waco, Tex.

Bibliothèque de L'Univérsité, 47 Rue Des Ecoles, Paris 5, France

Public Library, 2020 Seventh Ave. N., Birmingham, Ala.

Bodleian Library, Oxford, England

Boston Athenaeum Library, 101/2 Beacon St., Boston 8, Mass.

Boston College, Chestnut Hill 67, Mass.

Boston Public Library, History Dept., Copley Sq., Boston 17, Mass.

Boston University, College of Liberal Arts Library, 725 Commonwealth Ave., Boston 15, Mass.

Library, Bowdoin College, Brunswick, Me.

Library, Bowling Green State University, Bowling Green, 0.

Bradley University Library, 1502 West Bradley Ave., Peoria 5, Ill.

Heber J. Grant Library, Brigham Young University, Provo, Utah

University of British Columbia, Vancouver 8, Canada

Brooklyn Public Lib., Central Ser., Grand Army Plaza, Brooklyn 38, New York

Brown University Lib., Providence 12, R. I.

Office International de Librairie, 184, Rue del'Hotel-des-Monnaies, Brussels, Belgium

Bryn Mawr College Lib., Bryn Mawr, Pa.

Ellen Clarke Bertrand Lib., Bucknell University, Lewisburg, Pa.

Ku-Egyetemi Konyvtar, Egyetem-U. 10, Budapest V., Hungary

California Academy of Sciences Lib., Golden Gate Park, San Francisco 18, Calif.

University of California, Gen. Lib., Berkeley 4, Calif.

University of California, Dept. of Geography, Berkeley 4, Calif.

Lib., University of California, Davis, Calif.

Santa Barbara College Lib., University of California, Goleta, Calif.

University of California, Lib., 405 Hilgard Ave., Los Angeles 24, Calif.

University of California Lib., Riverside, Calif.

California State Lib., Sacramento 14, Calif.

Chicago Public Lib., 78 East Washington, Chicago 2, Ill.

University of Chicago, Harper M 22, Chicago 37, Ill.

Public Lib., 629 Vine St., Cincinnati 2, 0.

Gen. Lib., University of Cincinnati, Cincinnati, 0.

Public Lib., 325 Superior Ave., Cleveland 14, 0.

Colorado College Lib., Colorado Springs, Colo.

University of Colorado Lib., Boulder, Colo.

Columbia University Lib., 535 West 114th St., New York 27, New York

Connecticut State Lib., Hartford, Conn.

University of Connecticut Lib., Storrs, Conn.

Kongel. Bibliothek, Christians Brygge 8, Copenhagen K, Denmark

Cornell University Lib., Ithaca, New York

Cossitt Ref. Lib., 33 South Front St., Memphis 3, Tenn.

Dallas Public Lib., 1926 Commerce St., Dallas 1, Tex.

Dartmouth College, Baker Lib., Hanover, N.H.

Denver Public Lib., Civic Center, Denver 2, Colo.

University of Denver Lib., Denver 10, Colo.

Detroit Public Lib., 5201 Woodward Ave., Detroit 2, Mich.

University of Detroit Lib., 4001 W. McNichols Rd., Detroit 21, Mich. 
Duke University Lib., Durham, N.C.

Eastern Michigan University Lib., Ypsilanti, Mich.

Enoch Pratt Free Lib., 400 Cathedral St., Baltimore 1, Md.

Erskine College, Due West, S.C.

Florida Presbyterian College Lib., St. Petersburg, Fla.

Florida State Lib., Supreme Court Bldg., Tallahassee, Fla.

Florida State University Lib., Tallahassee, Fla.

University of Florida Lib., Gainesville, Fla.

Fort Hays Kansas State College Lib., Hays, Kan.

Fort Worth Public Lib., Fort Worth 2, Tex.

Franklin \& Marshall College Lib., Lancaster, Pa.

Fresno State College Lib., Fresno, Calif.

Funk \& Wagnalls Co. Lib., 153 East 24th St., New York 10, New York

Gaston Co. Public Lib., Gastonia, N.C.

Georgia Institute of Technology, Library, Atlanta 13, Ga.

Georgia State College of Bus. Adm. Lib., 33 Gilmer St., S. E., Atlanta 3, Ga.

University of Georgia Lib., Athens, Ga.

Grinnell College Lib., Grinnell, Iowa

Grosvenor Lib., 383 Franklin St., Buffalo 2, New York

Gumperts Aktie Bolag, Goeteborg, Sweden

Hans Hartinger Nachf., Podbielskieallee 40, Berlin-Dahlem, Germany

Harvard University Lib., Cambridge 38, Mass.

University of Hawaii Lib., Honolulu 14, Hawaii

Henry E. Huntington Lib. \& Art Gallery, San Marino, Calif.

Idaho State College, Pocatello, Idaho

Illinois Institute of Technology, Chicago 16, Ill.

University of Illinois Lib., Urbana, Ill.

Indiana State Lib., 140 N. Senate Ave., Indianapolis 4, Ind.

Indiana University Lib., Bloomington, Ind.

State Historical Society of Iowa City, Iowa

State University of Iowa Lib., Iowa City, Iowa

Public Lib., Market Square, Johannesburg, South Africa

John Carroll University Lib., Cleveland 18, 0.

Johns Hopkins University Lib., Baltimore 15, Md.

Joint University Lib., Nashville, Tenn.

Public Lib., Kansas City 6, Mo.

Kansas State College Lib., Manhattan, Kan.

University of Kansas Lib., Lawrence, Kan.

Kemink Zoon's Baekhandel, Domstraat 1-3, Utrecht, Holland.

Kent State University Lib., Kent, 0.

University of Kentucky Lib., Lexington, Ky.

Kirberger \& Kesper Oude Turfmarkt 153, Amsterdam, Holland

Kungl. Biblioteket, Stockholm 5, Sweden

Bibliothèque Générale, Université Laval, Quebec, Canada

Lehigh University Lib., Bethlehem, Pa.

University Lib., Rapenburg 70-74, Leiden, The Netherlands

Academy of Sciences USSR Lib., Birshevaia Liniia 1, Leningrad 164, USSR 
Library of Congress, Washington 25, D.C.

City Lib., Lincoln, Nebr.

Lincoln Lib., Shippensburg, Pa.

Los Angeles City College Lib., Los Angeles 29, Calif.

Los Angeles Public Lib., Los Angeles 17, Calif.

Los Angeles State College Lib., Los Angeles 32, Calif.

Louisiana State University Lib., Baton Rouge 3, La.

Free Public Lib., Louisville 3, Ky.

Bibliothèque de L'Université, Place Mgr. Ladeuze, Louvain, Belgium

A. B. Lumdequistska Bokhandeln, Uppsala, Sweden

Maine Historical Society, 485 Congress St., Portland, Me.

University of Manitoba Lib., Winnipeg, Manitoba, Canada

Bibliothèque de l'Université Aix Marseille, Aix en Provence/BDR, France

Marshall College Lib., Huntington 1, W.Va.

University of Maryland Lib., College Park, Md.

Memorial University Lib., St. John's, Newfoundland, Canada

Universidad N. A. Mexico, Biblioteca Central, Ciudad Universitaria, D. F., Mexico

Miami Public Lib., Miami 32, Fla.

Miami University Lib., Oxford, 0.

Michigan Historical Commission, Lansing, Mich.

Michigan State Lib., Lansing, Mich.

Michigan State University Lib., East Lansing, Mich.

University of Michigan Lib., Ann Arbor, Mich.

Middle Tennessee State College Lib., Murfreesboro, Tenn.

Milwaukee Public Lib., Milwaukee 3, Wis.

Minneapolis Public Lib., Minneapolis 3, Minn.

University of Minnesota Lib., Minneapolis 14, Minn.

University of Mississippi Lib., University, Miss.

Missouri State Lib., Jefferson City, Mo.

University of Missouri Lib., Columbia, Mo.

Tec. Proc. Lib., Montana State University, Missoula, Mont.

Historical Society of Montana, Helena, Mont.

Bibliotheka Akademii, Nauk SSSR, UL Frunze 11, Moscow 19, USSR

Filial Biblioteki, Akademii Nauk, Baltkjsky Poselok 42-B, Moscow D-219, USSR

Filial Biblioteki, Akademii Nauk SSSR, Baltijska ja ul., 14, Moscow D-219, USSR

Vses. Gosbiblioteka, Inoliteraturi, Glavpochta p/ja 964, Moscow, USSR

Gosudarstvennaia (Lenin) Biblioteka SSSR, Otdel Mezhdunarodnogo Knigoobmena,

U1. Kalinina 3, Moscow G-19, USSR

Universitätsbibliothek, 13B München 22, Geschwister-Scholl-Platz 1, Germany

Murray State College Lib., Murray, Ky.

National Geographic Society Lib., Washington 6, D.C.

Nebraska State Historical Society, Lincoln 8, Nebr.

University of Nebraska Lib., Lincoln 8, Nebr.

Nevada Historical Society, Reno, Nev.

University of Nevada Lib., Reno, Nev.

Newark Public Lib., Newark 1, N.J.

Newberry Lib., Chicago 10, Ill. 
New Century Publishers, Inc., 832 Broadway, New York, New York

Free Public Lib., New Haven 10, Conn.

New Mexico A \& M University Lib., University Park, N.M.

University of New Mexico Lib., Albuquerque, N.M.

New Mexico Western College Lib., Silver City, N.M.

New South Wales Public Lib., Sidney, Australia

New York Public Lib., New York 18, N.Y.

New York State Lib., Albany 1, N.Y.

New York University Washington Square Lib., New York 3, N.Y.

Niedersächsische Staats-Universitätsbibliothek, Göttingen, Prinzenstr. 1, Germany

Public Lib., Norfolk 10, Va.

University of North Carolina Lib., Chapel Hill, N.C.

Women's College Lib., University of North Carolina, Greensboro, N.C.

University of North Dakota Lib., Grand Forks, N.Dak.

Northern Illinois University Lib., Dekalb, Ill.

Northwestern University Lib., Evanston, Ill.

University of Notre Dame Lib., Notre Dame, Ind.

Public Lib., Oakland 12, Calif.

Occidental College Lib., Los Angeles 41, Calif.

Ohio State Lib., Columbus 15, O.

Ohio State University Lib., Columbus 10, 0.

Oklahoma City Lib., Oklahoma City 2, Okla.

Oklahoma State University Lib., Stillwater, Okla.

University of Oklahoma Lib., Norman, Okla.

Oregon State College Lib., Corvallis, Ore.

Oregon State Lib., Salem, Ore.

University of Oregon Lib., Eugene, Ore.

Board on Geographical Names, No. 8, G. M. Monroe, Sec., Ottawa, Canada

Geographical Branch Lib., 75 Elgin Bldg., Ottawa 4, Canada

National Museum of Canada Lib., Ottawa 4, Canada

Peabody Museum Lib., Salem, Mass.

Historical Society of Pennsylvania, Philadelphia 7, Pa.

Pennsylvania State Lib., Harrisburg, Pa.

Pennsylvania State University Lib., University Park, Pa.

Philadelphia Free Lib., Middle City West Dist., Philadelphia 3, Pa.

University of Pennsylvania Lib., Philadelphia 4, Pa.

University of Pittsburgh Lib., Pittsburgh 13, Pa.

Library Association of Portland, Portland 5, Ore.

Pratt Institute Lib., Brooklyn 5, N.Y.

Princeton University Lib., Princeton, N.J.

University of Puerto Rico Lib., Rio Piedras, Puerto Rico

Purdue University Lib., Lafayette, Ind.

Queens Borough Public Lib., 89-14 Parsons Blvd., Jamaica 2, N.Y.

Queens College, Serials Librarian, Paul Klapper Library, Flushing 67, N.Y.

Queens University Lib., Kingston, Ontario, Canada

Rand McNally Lib., Chicago 80, $\mathrm{Ill}$.

University of Rhode Island Lib., Kingston, R.I. 
Rice Institute Fondren Lib., Houston 1, Tex.

William Marsh Rice Univ., Houston, Tex.

P. K. W. Z. "Ruch" 3917, Aleje Jerozolimskie 119, Warzawa, Poland

Rutgers University Lib., New Brunswick, N.J.

St. Louis Public Lib., St. Louis 3, Mo.

Biblioteca De La Facultad De Filosofia, Plaza De La Anaya, Salamanca, Spain Genealogical Society, 80 N. Main St., Salt Lake City 1, Utah

San Diego Public Lib., San Diego 1, Calif.

Sandoz Library, Ellsworth, Nebr.

Public Lib., San Francisco 2, Calif.

San Jose City College Lib., San Jose, Calif.

San Jose State College, Joyce Backus, Librarian, San Jose 14, Calif.

College of San Mateo Lib., San Mateo, Calif.

Saskatchewan Archives, John H. Archer, Room 275, Legislative Bldg., Regina,

Saskatchewan, Canada

University of Saskatchewan Lib., Regina, Saskatchewan, Canada

Seattle Public Lib., Seattle 4, Wash.

Smithsonian Institution Lib., Washington 25, D.C.

University of South Carolina Lib., Columbia 1, S.C.

South Dakota State College Lib., State College Station, S.Dak.

University of South Dakota Lib., Vermillion, S.Dak.

University of Southern California Lib., Los Angeles 7, Calif.

Southern Illinois University Lib., Carbondale, Ill.

Southwest Museum Lib., Los Angeles 42, Calif.

Central Lib., Southern Illinois University, Edwardsville, Ill.

Spokane Public Lib., Spokane 4, Wash.

Stanford University Lib., Stanford, Calif.

Humanistika Biblioteket, Stockholm Hoegskola, Stockholm 6, Sweden

Syracuse University Lib., Syracuse 10, N.Y.

Temple University Lib., Philadelphia 22, Pa.

Tennessee State Lib. \& Archives, Nashville 3, Tenn.

University of Tennessee Lib., Knoxville 16, Tenn.

A. \&. M. College of Texas, College Station, Tex.

Texas Christian University Lib., Forth Wort 9, Tex.

Texas Woman's University Lib., Denton, Tex.

Texas Technological College Lib., Lubbock, Tex.

University of Texas Lib., Austin 12, Tex.

Toledo Public Lib., Toledo 2, 0.

University of Toledo Lib., Toledo, 0.

Gerhard Trenkle, OHG, Literatur Import, Planegg Kreuzwinkel, Germany

Trinity University Lib., San Antonio, Tex.

University of Tulsa Lib., Tulsa, Okla.

United Nations Lib., New York, N.Y.

U. S. Air Force Academy, Colorado Springs, Colo.

U. S. Army Map Service, Washington 16, D.C.

U. S. Coast \& Geodetic Survey Lib., Washington 25, D.C.

Office of Geography Lib., U. S. Dept. of Interior, Washington 25, D.C. 
U. S. Geological Survey Lib., Washington 25, D.C.

U. S. Navy Hydrographic Office, Suitland, Md.

University College Lib., London W.C. 1, England

Utah State Agric. University Lib., Logan, Utah

University of Utah, Salt Lake City, Utah

Vancouver Public Lib., Vancouver, B.C., Canada

Vassar College, Poughkeepsie, N.Y.

Vermont Historical Soc., Montpelier, Ver.

University of Vermont Lib., Burlington, Ver.

Provincial Lib., Victoria, B.C., Canada

Virginia Historical Society, Richmond 21, Va.

Virginia State Lib., Richmond 19, Va.

University of Virginia Lib., Charlottesville, Va.

Waco Public Lib., Waco, Tex.

Washington State University Lib., Pullman, Wash.

University of Washington Lib., Seattle 5, Wash.

Washington University Lib., St. Louis 5, Mo.

Wayne State University Lib., Detroit 2, Mich.

Western Reserve University Lib., Cleveland 6, 0.

West Texas State College Lib., Canyon, Tex.

West Virginia University Lib., Morgantown, W.Va.

West Virginia Wesleyan College Lib., Buckhannon, W.Va.

Wheaton College Lib., Wheaton, Ill.

University of Wichita Lib., Wichita 14, Kan.

College of William \& Mary Lib., Williamsburg, Va.

Winthrop College Lib., Rock Hill, S. Car.

Wisconsin State Historical Society, Madison 6, Wis.

University of Wisconsin Lib., Madison 6, Wis.

University of Wisconsin, Kenwood Campus Lib., Milwaukee 11, Wis.

University of Witwatersrand Lib., Johannesburg, South Africa

World Publishing Co., David B. Guralnik, Cleveland 2, 0.

University of Wyoming Lib., Laramie, Wyo.

Yale University Lib., New Haven, Conn.

Youngstown University Lib., Youngstown, $\mathrm{O}$. 